\title{
El estrés en personal sanitario hospitalario; estado actual
}

\section{The stress in the hospital sanitary personnel; current status}

\author{
Jose Ignacio Aguado Martín 1,4, Ainhoa Bátiz Cano ${ }^{2,4,}$ Salomé Quintana Pérez ${ }^{3,4}$ \\ 1. Unidad Docente de Medicina del Trabajo. La Rioja. Mutua Universal. Logroño. \\ 2. Unidad Docente de Medicina del Trabajo. La Rioja. Hospital San Pedro .Logroño. \\ 3. Unidad Docente de Medicina del Trabajo. Asturias. Hospital Universitario Central de Asturias. \\ 4. Unidad Docente de Medicina del Trabajo. Madrid. España
}

Recibido: 28-03-13

Aceptado: 20-05-13

\section{Correspondencia}

Jose Ignacio Aguado Martin

Servicio de Prevención de Riesgos Laborales

Hospital San Pedro

C/ Piqueras 98

CP 26007 Logroño (La Rioja) España.

Tfno: 941298390

E-mail: jiaguado@riojasalud.es

Resumen

Introducción: Los riesgos psicosociales y en particular el estrés se han convertido en uno de los principales problemas para la salud y la seguridad laboral. Se ha realizado una revisión de la literatura científica actual sobre el estrés debido a su importancia como riesgo emergente, y la repercusión de este problema en la productividad y el deterioro de la calidad de vida en el personal sanitario que desarrolla su actividad profesional en medio hospitalario, así como intentar identificar los principales métodos de evaluación del estrés actualmente utilizados.

Objetivos: Conocer el estado actual del estrés en el personal hospitalario e identificar los principales métodos de evaluación.

Metodología: Se realizo una búsqueda bibliográfica exploratoria utilizando las bases de datos Medline, Embase, Scirus, Wok, Psicoinfo, OSH update, Cisdoc, IBECS y LILACS, así como en repositorios como Dialnet, SciELO y Google académico. El periodo de estudio de la bibliografía publicada fue el comprendido entre el año 2009 y 2012 realizándose la última búsqueda el 10 de diciembre de 2012.

Resultados: Los niveles de estrés encontrados en los artículos revisados fueron moderados-altos, con diferencias entre las distintas áreas y especialidades. Factores individuales tanto objetivos como subjetivos parecen influir en los grados de estrés. Existe diversidad de métodos utilizados para la evaluación del estrés, la mayoría tienen un nivel de fiabilidad elevado. La producción bibliográfica encontrada es mayor en Asia y Europa.

Conclusiones: Los niveles de estrés son elevados en el personal sanitario del hospital ,sobre todo en algunas areas y especialidades, existen una serie de moderadores del grado de estrés como los factores individuales y el ambiente físico y no existe un consenso internacional en el método utilizado para su evaluación.

Med Segur Trab (Internet) 2013; 59 (231) 259-275

Palabras Clave: Estrés; Estrés psicológico; Personal del bospital; Evaluación; Estrés ocupacional. 


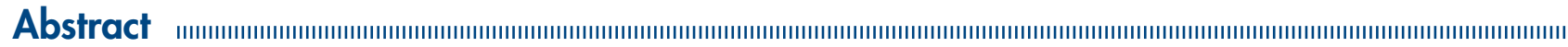

Introduction: Psychosocial risks and stress in particular have become one of the major problems for health and safety. There has been a review of the current scientific literature on stress due to its importance as an emerging risk, and the impact of this problem on productivity and impaired quality of life in personnel hospital and try to identify the main stress assessment methods currently used.

Objective: To determine the current state of stress in personnel hospital and identify the main evaluation methods.

Methods: A Bibliography review using Medline, Embase, Scirus, Wok, Psicoinfo, OSH update, CISDOC IBECS and LILACS as well as repositories as Dialnet, SciELO and Google Scholar. The study period of the published literature was between 2009 and 2012 carried the last search December 10, 2012.

Results: Stress levels found in the articles reviewed were moderate-high, with differences between different areas and specialties. Individual factors both objective and subjective appear to influence the degree of stress. There is variety of methods used for the evaluation of stress; most have a high reliability level. The literature found production is higher in Asia and Europe.

Conclusion: Stress levels are high in the sanitary personnel hospital, especially in some areas and specialties. There are a number of moderators of the degree of stress as individual factors and the physical environment, and there is no international consensus on the method used for evaluation.

Med Segur Trab (Internet) 2013; 59 (231) 259-275

Key Words: Stress; Psychological Stress; Hospital Personnel; Evaluation; Occupational stress. 


\section{INTRODUCCIÓN}

A lo largo del siglo XX los riesgos psicosociales se han convertido en un fenómeno relevante y reconocido socialmente, vinculado al trabajo y a la actividad laboral.

Tradicionalmente los servicios de prevención de riesgos laborales abordaban temas relacionados con la seguridad, higiene y ergonomía, mientras que hasta no hace mucho, los factores psicosociales no eran considerados como una prioridad.

En la 9. ${ }^{a}$ reunión del comité mixto formado por la Organización Internacional del Trabajo (OIT) y la Organización Mundial de la Salud (OMS), celebrada en Ginebra en 1984, abordan entre sus temas del orden del día, la identificación y el control de los factores psicosociales nocivos en el trabajo. La tendencia, a partir de ese momento fue que además de tener en cuenta los factores de riesgo físicos, químicos y biológicos del medio ambiente, se considerasen también los factores psicosociales inherentes al entorno laboral como un factor de riesgo y como estos podían influir en el bienestar físico y mental del trabajador.

En este mismo año, la OIT publica uno de los primeros documentos oficiales e internacionales que abordan dicho tema: "Los Factores psicosociales en el Trabajo: Reconocimiento y Control”. A partir de 1999, el Instituto Nacional de Seguridad e Higiene en el Trabajo (INSHT) incorpora los factores psicosociales en sus Encuestas sobre Condiciones de Trabajo en nuestro país.

Los riesgos psicosociales más ampliamente reconocidos y sobre los que se mantiene un relativo consenso son el estrés, violencia en el trabajo, acoso laboral o moobing, acoso sexual, inseguridad laboral y Burnout o desgaste profesional. (BMJiménez), aunque hay que tener en cuenta que otros factores del ámbito psicosocial, como la intensidad laboral, el envejecimiento de la población trabajadora (OIT Recomendación $\mathrm{N}^{\mathrm{o}}$ 162), la irregularidad de los tiempos de Trabajo (Brun y Milczarek, 2007), el conflicto trabajofamilia (Greenhouse y Beutel) y el trabajo emocional (Arlie R. Hochschild), también constituyen factores de riesgo importantes para la salud, aunque hasta el momento hayan podido ser menos estudiados.

El estrés, como uno de los factores psicosociales más ampliamente estudiados, es un término que procede de la física y de la arquitectura, entendiéndose como la fuerza que soporta un objeto hasta poder llegar a deformarlo o romperlo.

El primero que importó este término a la psicología fue el húngaro Hans Selye (1907-1982), con el nombre de Síndrome de Adaptación General (SAG) y lo definió como una respuesta no específica del cuerpo frente a cualquier demanda, de forma que si su duración es suficientemente prolongada, el organismo puede entrar en una etapa de agotamiento.

Uno de los principales problemas que nos encontramos a la hora de estudiar el estrés, es la dificultad que revierte para estimarlo, por lo que varios investigadores han postulado diversos modelos teóricos que buscan describir de una manera objetiva este fenómeno, y así nos encontramos con el Modelo Demanda-Control (KaraseK 1979), Modelo vitamínico (Warr 1987), Modelo de ajuste Persona-Entorno (Harrison 1987), Modelo OSI (Occupational stress indicator) (Copper, Sloan y Willimas 1988), y Modelos holísticos de Nelson y Simmons (2003).

La OMS define el estrés laboral como un patrón de reacciones psicológicas, cognitivas y conductuales que se dan cuando los trabajadores se enfrentan a exigencias ocupacionales que no se corresponden con su nivel de conocimiento, destreza o habilidades.

En cuanto a los principales estresores laborales, y aunque existen muchas formas de clasificación, podíamos considerar como más relevantes, el ambiente físico, demandas del propio trabajo, estructura de la organización, relaciones interpersonales, inseguridad laboral, organización del trabajo, conflicto de roles y factores externos al trabajo (Peiro 1999). 
Según la OIT, el 30\% de la población activa sufre estrés laboral, siendo esta cifra mayor en los países en vías de desarrollo. En Europa, la Tercera Encuesta sobre Condiciones de Trabajo de la Fundación Europea para la Mejora de las Condiciones de Vida y Trabajo (EUROFOUND 2000) identifica el estrés como la segunda enfermedad más frecuente. Se puede decir que el estrés laboral es uno de los principales problemas para la salud y la seguridad en el trabajo que nos encontramos en Europa, y casi el 25\% de los trabajadores se ven afectados por él y según la Agencia Europea de Seguridad y Salud en el Trabajo (EU- OSHA), entre un 50-60 \% de las bajas laborales se encuentran relacionadas con el estrés laboral.

Además de que el estrés puede provocar enfermedades y sufrimiento en las personas, la repercusión de este problema en término de pérdida de productividad, enfermedad y deterioro de la calidad de vida es muy alta, contribuir a la aparición de otros problemas de salud laboral, como frecuentemente son los trastornos musculo-esqueléticos y puede poner en peligro la seguridad en el lugar de trabajo.

Por tanto, el estrés laboral es uno de los riesgos psicosociales más importantes en nuestra sociedad y entre los principales retos relacionados con su estudio, deberán encaminarse hacia la identificación de nuevos estresores presentes en el entorno laboral y a la necesidad de atender simultáneamente el bienestar del empleado y de la organización.

Muchas profesiones se encuentran sometidas a niveles elevados de estrés, y dentro de ellas, el ámbito sanitario, y más en concreto los médicos y enfermeras, son considerados como uno de los sectores profesionales más expuestos niveles elevados de estrés, tanto de forma puntual como mantenida. (Adán Mingote) ${ }^{1,2,3}$.

El objetivo principal de este estudio se centra en conocer el estado actual del estrés en el personal sanitario hospitalario, médicos y enfermeras, así como identificar los principales métodos de evaluación del estrés que actualmente se encuentran utilizando.

\section{MATERIAL Y MÉTODOS}

Se realiza una revisión bibliográfica de la literatura científica publicada durante el periodo 2009-2012, abordando el estrés en personal sanitario que desarrolla su trabajo en el medio hospitalario. La búsqueda se realizó entre el 6 de noviembre de 2012 y el 10 de diciembre de 2012, consultando las siguientes bases de datos.

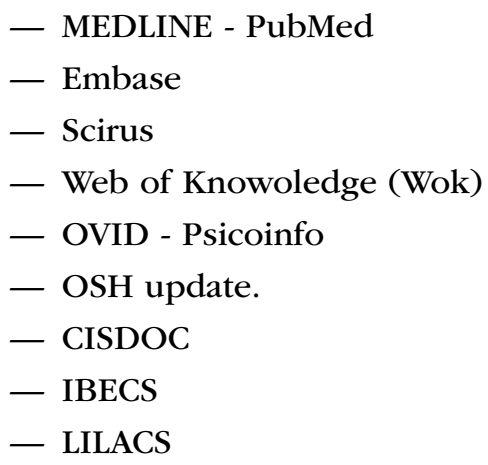

La búsqueda bibliográfica en bases de datos, fue completada con los resultados obtenidos de repositorios como Dialnet, SciELO y Google académico.

Para realizar la búsqueda bibliográfica utilizaron descriptores y términos libre DeCS y MeSH, estableciéndose la siguiente estrategia de búsqueda:

- Términos libres: work stress, occupational stress, job stress, work related stress, stress, assessment, hospital, hospital worker, evaluacion de riesgos y estrés laboral 
- Descriptores: personnel hospital, risk assessment, stress psychological, evaluación, evaluation, burnout

Dado que en el DeCS y MeSH, el término "Burnout" se encuentra clasificado dentro de la estructura jerárquica del término "estrés laboral" y otras bases de datos los consideran como términos sinónimos, se optó por utilizar la estrategia "estrés = work stress NOT Burnout" para obtener una selección de artículos más precisa.

Una vez obtenida la colección bibliográfica, se realizó un análisis de pertinencia cotejando la idoneidad de los artículos para el estudio a partir de la revisión de los títulos y el resumen de los mismos.

Posteriormente, a los artículos seleccionados, se les aplicó los siguientes criterios de inclusión y exclusión:

Criterios de Inclusión.

- Estudios en humanos de ambos sexos.

- Estudios de trabajadores activos.

- Estudios sobre el estrés laboral.

- Estudios en personal hospitalario.

- Escrito en inglés y castellano.

- Publicados en el periodo 2009-2012.

- Estudios con abstract disponible.

Criterios de exclusión.

- Estudios sobre el Burnout exclusivamente.

- Estudios de personal no sanitario.

— Estudios sobre estrés no relacionado con el ámbito laboral.

- Libros, protocolos, revisiones, editoriales, comunicaciones, o cartas al director, notas técnicas o a propósito de un caso.

\section{RESULTADOS}

A partir de la estrategia de búsqueda se obtuvo un total de 690 artículos. Una vez eliminados los duplicados, se procedió a un análisis de pertinencia y a aplicar los criterios de inclusión y exclusión, obteniéndose una colección final de 26 artículos que conforman la base del estudio (Tabla I). 


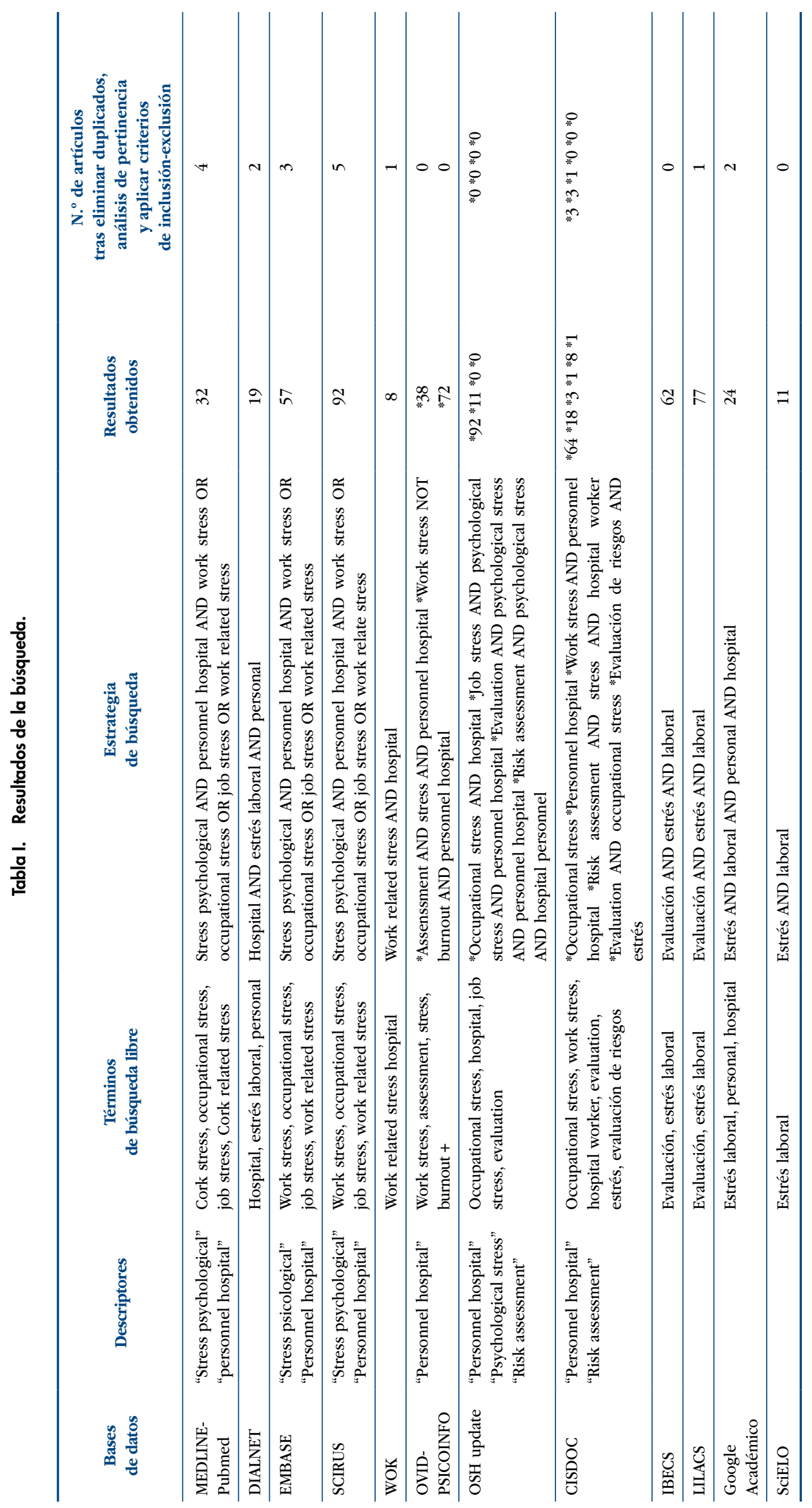


Todos los artículos encontrados se correspondían con estudios descriptivos transversales y en varios estudios la población supera los 1000 sujetos.

En cuanto a la distribución y tendencia temporal de las publicaciones, encontramos que el mayor número de artículos publicados se corresponden con 2009 y posteriormente presenta una tendencia decreciente progresivo y constante hasta el último año de estudio correspondiente a 2012, lo que parece indicar una disminución de interés progresivo en el estudio del estrés en el personal sanitario durante los últimos años (Tabla II).

Tabla II. Distribución de artículos por años.

\begin{tabular}{ccccc}
\hline Año & 2009 & 2010 & 2011 & 2012 \\
\hline $\mathrm{N}^{\circ}$ de artículos. & 11 & 8 & 6 & 1 \\
\hline
\end{tabular}

La producción científica más abundante se genera $\mathrm{n}$ grupos de investigación afincados en Asia con un total de 14 artículos (548\%), seguida de Europa con 6 publicaciones (23\%), uno de ellos realizado en España (Tabla III).

Tabla III. Distribución del número de artículos por países o áreas geográficas

\begin{tabular}{llc}
\hline \multicolumn{1}{c}{ País } & Zona geográfica & Numero de estudios \\
\hline Finlandia & Europa & 1 \\
Italia & Europa & 1 \\
Dinamarca & Europa & 1 \\
Irlanda & Europa & 1 \\
España & Europa & 1 \\
5 países europeos (Austria, Rumania, Italia, UK, Irlanda) & Europa & 1 \\
6 países (Holanda, Francia y Suecia, Japón, España, China) & & 1 \\
Costa Rica & Suramérica & 1 \\
México & Suramérica & 2 \\
Venezuela & Suramérica & 2 \\
Brasil & Suramérica & 1 \\
Japón & Asia & 3 \\
China & Asia & 2 \\
Taiwán & Asia & 1 \\
Mongolia & Asia & 1 \\
Irán & Asia & 2 \\
India & Asia & 1 \\
EEUU & Norte América & 2 \\
Jamaica & América \\
Jordania & África & 1 \\
\hline
\end{tabular}

Los trabajos realizados en personal de enfermería, representan el 46\%. El 39\% de los estudios se realizaron en trabajadores no sanitarios del ámbito de la salud y sólo un 15\% de estudios fueron realizados en médicos.

En cuanto a las principales características y resultados de los artículos estudiados, se representan en la Tabla IV, donde se resumen las principales peculiaridades cada uno en cuanto a título, autores, país donde se realiza el estudio, año en que se publica, tipo de estudio atendiendo al diseño, idioma en el que se publica, las principales características de la población estudiada, el material y método utilizados para el estudio y los principales resultados y conclusiones del estudio. 


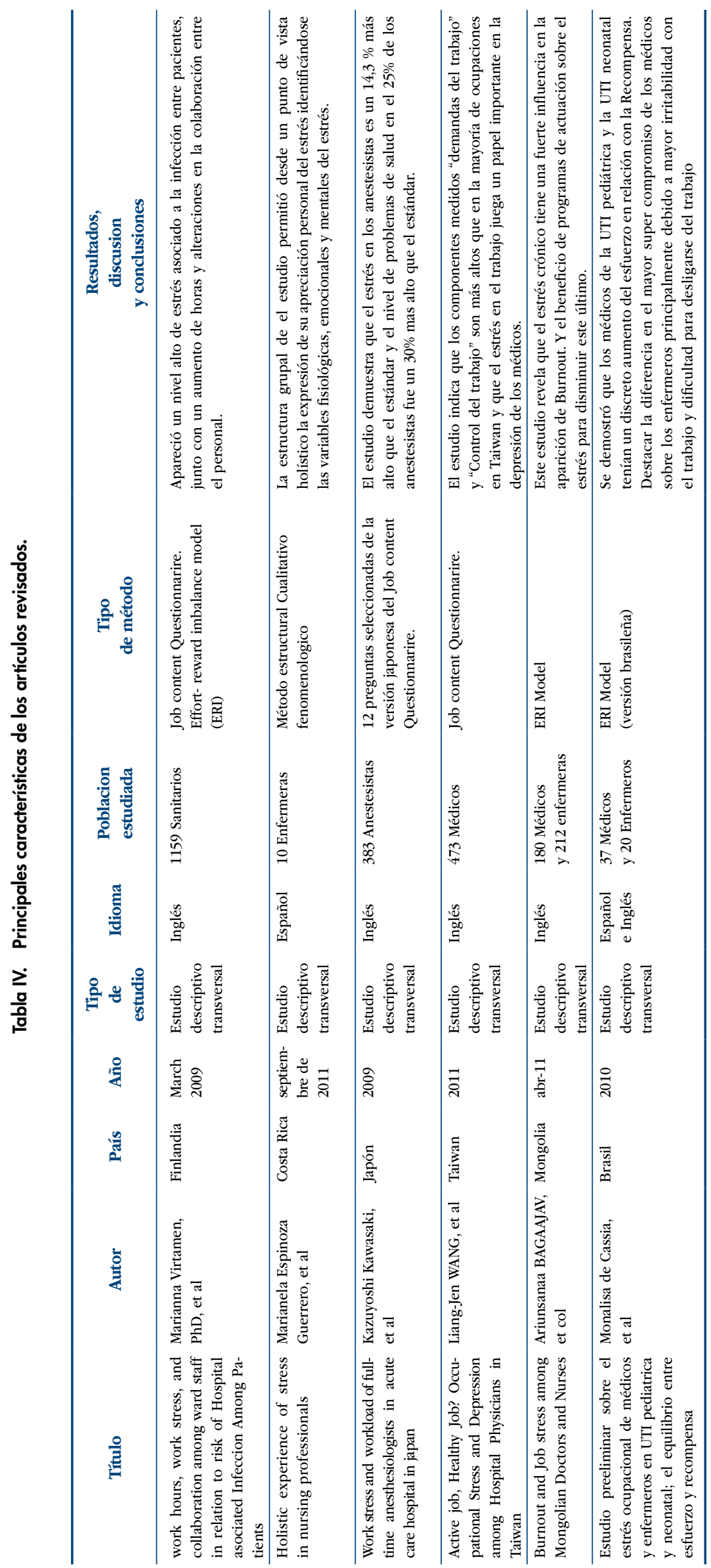

El estrés en personal sanitario hospitalario; estado actual 266 


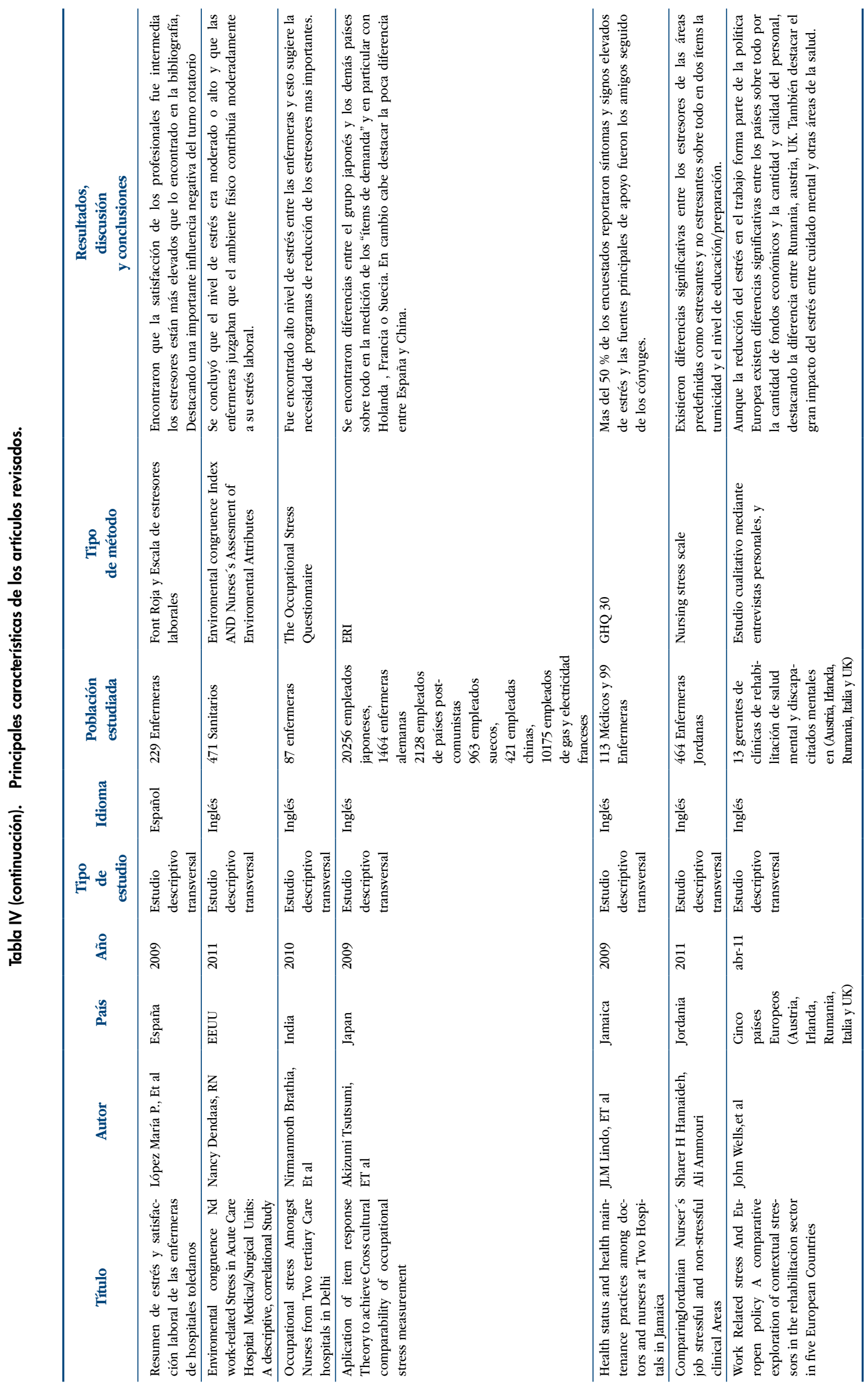




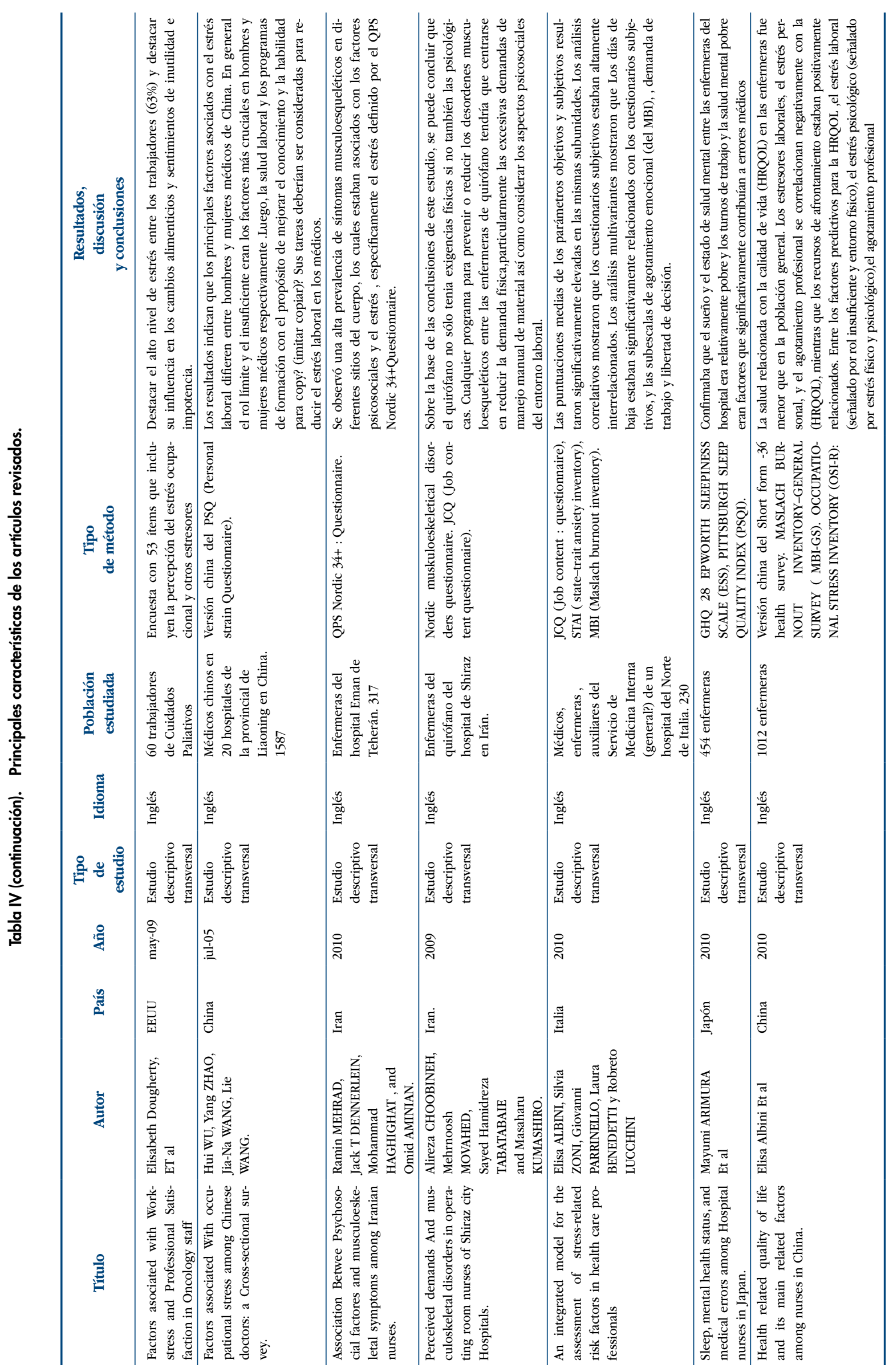




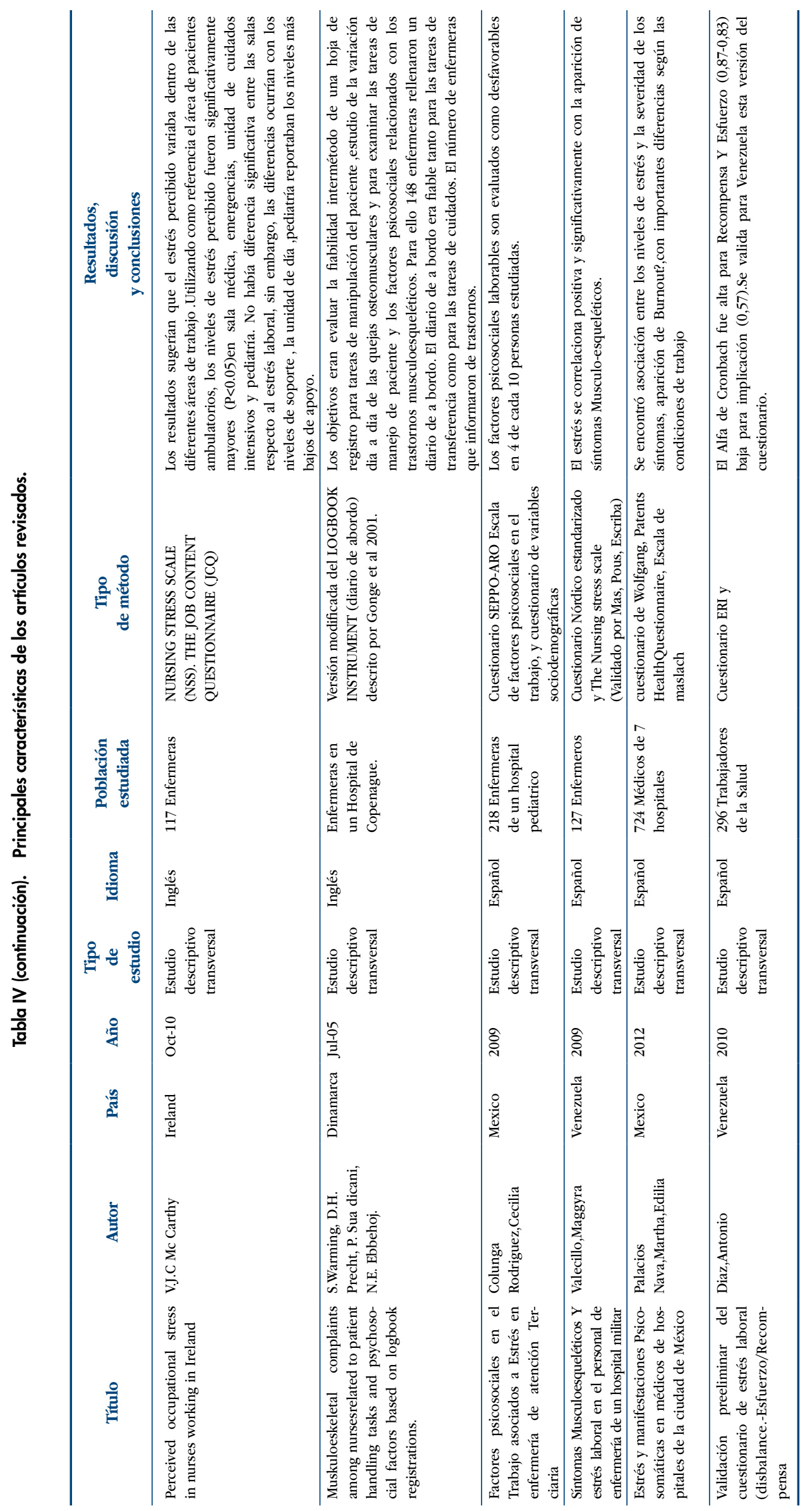

El estrés en personal sanitario hospitalario; estado actual 269 
Entre los principales resultados encontrados en artículos que evalúan de forma específica el grado de estrés en el personal sanitario, este se corresponde con un nivel moderado-elevado y parecen existir diferencias de nivel de estrés según las condiciones de trabajo, el área de estudio y el apoyo de los superiores y compañeros.

Algunos artículos señalan una correlación directa entre el estrés con la depresión, alteraciones psicosomáticas, lesiones musculo esqueléticas, así como con el Burnout.

Los factores individuales tanto objetivos como subjetivos tales como la percepción personal del estrés, las variables fisiológicas emocionales y mentales, el apoyo familiar y las demandas del propio trabajo, también parecen ser condicionantes e influir en el grado de estrés.

En el estudio realizado por Akizumi Et al, se compararon varios países utilizando escalas de esfuerzo-recompensa estandarizadas encontrándose diferencias particularmente significativas entre los resultados obtenidos en Japón y países de Europa como Holanda , Francia y Suecia en contraste con los resultados que comparan China , Japón y España, donde las diferencias son menos evidentes .

El conflicto de rol aparece en varios estudios como factor predisponente al estrés, así bien, la formación, las técnicas de afrontamiento se muestran como protectores. Se encontró moderada relación entre el espacio físico y el estrés entre enfermeras, y sólo se encontró un estudio realizado en España.

Uno de los estudios realizados en China, encontró diferencias entre profesionales de la salud masculinos y femeninos, en relación a los principales factores asociados al estrés, encontrándose las fronteras del rol, que valora el grado en que el individuo experimenta demandas de rol y lealtades conflictivas en su ambiente de trabajo, más comúnmente asociado al estrés en los varones. La insuficiencia de rol, que valora el grado en que el nivel de especialización, la educación, las habilidades y la experiencia del individuo se encuentran ajustadas a los requerimientos del trabajo y más asociado al estrés en las mujeres. Para este estudio se empleó una versión China del Personal Strain Questionnaire (PSQ).

Un modelo general lineal mostró que el PSQ estaba asociado con la siguiente secuencia; las fronteras de rol, la insuficiencia de rol, la responsabilidad, el soporte social, las técnicas de afrontamiento cognitivo-racionales, la relación médico-paciente y la sobrecarga de rol, esto en el caso de los hombres, mientras que en las mujeres las secuencia sería la siguiente: la insuficiencia de rol, las fronteras del rol, el soporte social, la responsabilidad, el autocuidado, la sobrecarga de rol, las enfermedades crónicas, y la relación médico paciente, por lo que en cuanto a los factores que afectan al estrés, parece encontrarse diferencias entre ambos sexos.

Finalmente el estudio concluye que la educación en salud ocupacional y los programas de entrenamiento ocupacional con el propósito de mejorar el conocimiento y las habilidades para adquirir técnicas de que permitan afrontar a cada individuo sus tareas, deberían tenerse en cuenta para disminuir el estrés ocupacional entre los médicos chinos. ${ }^{4}$

En cuanto a los métodos más utilizados para la evaluación del estrés, se encuentran descritos en la Tabla V, que además presenta las principales características de los mismos en cuanto a autoría, denominación del método, dimensiones que analiza y las principales ventajas e inconvenientes de cada uno de ellos. 
Tabla V. Tipos de métodos de evaluación del estrés

\begin{tabular}{|c|c|c|c|c|}
\hline Autor & Nombre & Dimensiones & $\begin{array}{l}\text { Núm. } \\
\text { de } \\
\text { ítems }\end{array}$ & Pros y contras \\
\hline \multirow[t]{2}{*}{ Seppo-Aro } & $\begin{array}{l}\text { Escala Sintomática de } \\
\text { estrés }\end{array}$ & $\begin{array}{l}\text { Condiciones de trabajo, carga de traba- } \\
\text { jo, contenido y características de la ta- } \\
\text { rea, exigencias laborales, papel del aca- } \\
\text { démico y desarrollo de la carrera, inte- } \\
\text { racción social, aspectos organizaciona- } \\
\text { les, remuneración del rendimiento. }\end{array}$ & 18 & $\begin{array}{l}\text { Alto respaldo estadístico. Valora el ni- } \\
\text { vel de estrés y las consecuencias so- } \\
\text { bre la salud. Alfa de Crombach }(0,83) \text {. }\end{array}$ \\
\hline & $\begin{array}{l}\text { Escala de valores } \\
\text { psicosociales }\end{array}$ & Similares a la anterior. & 47 & $\begin{array}{l}\text { Poco usada de forma internacional. } \\
\text { Alfa de Cronbach }(0,88) \text {. }\end{array}$ \\
\hline J.V. Jonson & $\begin{array}{l}\text { JCQ (job content } \\
\text { questionnarie) }\end{array}$ & $\begin{array}{l}\text { Inseguridad laboral, uso de habilida- } \\
\text { des, toma de decisiones, demanda de } \\
\text { trabajo, apoyo de compañeros, apoyo } \\
\text { supervisor. }\end{array}$ & & $\begin{array}{l}\text { Alta consistencia interna y validez in- } \\
\text { ternacional. Alfa de Cronbach }(0,84) \text {. } \\
\text { Desarrolla y añade a la escala de Ka- } \\
\text { rasek el apoyo social. }\end{array}$ \\
\hline J. Siegrist & $\begin{array}{l}\text { Cuestionario de } \\
\text { esfuerzo recompensa } \\
\text { (Effort-Reward } \\
\text { imbalance). }\end{array}$ & $\begin{array}{l}\text { Escala de esfuerzo, escala de recom- } \\
\text { pensa, implicaciones en el puesto. }\end{array}$ & $17+6$ & $\begin{array}{l}\text { Alto reconocimiento internacional. } \\
\text { Destaca la enfatización de las condi- } \\
\text { ciones del trabajo. Alfa de Crombach } \\
0,75 \text {. Relacióna la visión de costo } \\
\text { Beneficio del trabajo con el nivel de } \\
\text { estrés sufrido. }\end{array}$ \\
\hline $\begin{array}{l}\text { Pamela } \\
\text { Craytoft y } \\
\text { James G. } \\
\text { Anderson }\end{array}$ & Nursing Stress Scale. & $\begin{array}{l}\text { Ambiente físico, ambiente psicológico, } \\
\text { ambiente social. }\end{array}$ & 34 & $\begin{array}{l}\text { Ampliamente utilizada en el mundo } \\
\text { anglosajón, validada para España, es- } \\
\text { tudia de forma específica las condi- } \\
\text { ciones de trabajo de las enfermeras. }\end{array}$ \\
\hline Wolfgang & $\begin{array}{l}\text { Cuestionario IEPS } \\
\text { de wolfgag (Health } \\
\text { profesion stress } \\
\text { questionarie). }\end{array}$ & $\begin{array}{l}\text { Reconocimiento profesional, responsa- } \\
\text { bilidad, conflictos de trabajo, incerti- } \\
\text { dumbre profesional. }\end{array}$ & 30 & $\begin{array}{l}\text { Escala que se utiliza de forma espe- } \\
\text { cífica para el estrés Sanitario. Alfa de } \\
\text { Crombach que varía entre } 0,83-0,91 \text {, } \\
\text { según estudios. }\end{array}$ \\
\hline Kronke Etal & $\begin{array}{l}\text { PHQ-1S Patients } \\
\text { Health Questionnarie } \\
\text { (Kronke Etal). }\end{array}$ & $\begin{array}{l}\text { Investiga los síntomas somáticos de de- } \\
\text { presión y ansiedad provocados por el } \\
\text { estrés. }\end{array}$ & 15 & $\begin{array}{l}\text { Alfa de Crombach 0,83-0,75. Validado } \\
\text { en España. }\end{array}$ \\
\hline Golberg & GHQ & $\begin{array}{l}\text { Cuestionario de Salud general autoperci- } \\
\text { bida que incluye alteraciones mentales. }\end{array}$ & $\begin{array}{l}\text { Distintas } \\
\text { versiones } \\
\text { e ítems. }\end{array}$ & $\begin{array}{l}\text { Ampliamente utilizada y validada } \\
\text { pero no específico de riesgos psico- } \\
\text { sociales. }\end{array}$ \\
\hline $\begin{array}{l}\text { Aranaz J., } \\
\text { Miro J. }\end{array}$ & $\begin{array}{l}\text { Cuestionario Font } \\
\text { Roja de satisfacción } \\
\text { laboral. }\end{array}$ & $\begin{array}{l}\text { Satisfacción, tensión, competencia, pre- } \\
\text { sión, promoción profesional, relación } \\
\text { interprofesional, relación con compañe- } \\
\text { ros, relación con superiores, caracterís- } \\
\text { ticas extrínsecas del estatus, monotonía } \\
\text { laboral. }\end{array}$ & & $\begin{array}{l}\text { Ampliamente usado en España, mide } \\
\text { sólo satisfacción laboral. }\end{array}$ \\
\hline $\begin{array}{l}\text { Facilites } \\
\text { Guidelines } \\
2006\end{array}$ & $\begin{array}{l}\text { Enviromental } \\
\text { Congruence index. } \\
\text { Nurses Assessment } \\
\text { of Enviromental. } \\
\end{array}$ & $\begin{array}{l}\text { Evalúa el ambiente físico que interactúa } \\
\text { con la enfermera. }\end{array}$ & $35+40$ & $\begin{array}{l}\text { Tras entrevista a las enfermeras eva- } \\
\text { lúa el ambiente físico como adecuado } \\
\text { o no según los aspectos inherentes al } \\
\text { trabajo. }\end{array}$ \\
\hline $\begin{array}{l}\text { Barrantes } \\
2000\end{array}$ & $\begin{array}{l}\text { Método estructural } \\
\text { cualitativo } \\
\text { fenomenológico. }\end{array}$ & $\begin{array}{l}\text { Intervención grupal y evaluación cuali- } \\
\text { tativa de los aprendizajes. }\end{array}$ & & $\begin{array}{l}\text { Tras entrevistas a las enfermeras de } \\
\text { forma grupal se ponen de manifiesto } \\
\text { sus apreciaciones y se intenta actuar } \\
\text { para mejorar su afrontamiento. }\end{array}$ \\
\hline $\begin{array}{l}\text { Psychological } \\
\text { Assessment } \\
\text { Inc. }\end{array}$ & $\begin{array}{l}\text { Osi-R (Occupational } \\
\text { Stress Inventory - } \\
\text { Revised Edition TM) }\end{array}$ & $\begin{array}{l}\text { Tres subescalas: ORQ: Occupational } \\
\text { stress. PSQ: Psychological strain. PRQ: } \\
\text { Coping resources. }\end{array}$ & & $\begin{array}{l}\text { Escala muy completa que estudia tres } \\
\text { dimensiones importantes de estrés } \\
\text { laboral. }\end{array}$ \\
\hline \multirow[t]{2}{*}{ Resources } & Loogbook Instrument & $\begin{array}{l}\text { Relación de factores estresantes y alte- } \\
\text { raciones musculoesqueléticas. }\end{array}$ & & $\begin{array}{l}\text { Diario de a bordo donde las enferme- } \\
\text { dades anotan la posible relación cau- } \\
\text { sal de situaciones con daño muscular. }\end{array}$ \\
\hline & & & & $\begin{array}{l}\text { En psicometría, el Alfa de Cronbach } \\
\text { es un coeficiente que sirve para me- } \\
\text { dir la fiabilidad de una escala de me- } \\
\text { dida, y cuya denominación Alfa fue } \\
\text { realizada por Cronbach en } 1951 \text { (1), } \\
\text { aunque sus orígenes se encuentran } \\
\text { en los trabajos de Hoyt (1941) (2) y } \\
\text { de Guttman (1945) (3). }\end{array}$ \\
\hline
\end{tabular}




\section{DISCUSIÓN}

La mayor parte de los autores coinciden en que los niveles de estrés a los que se encuentran sometidos los trabajadores sanitarios del ámbito hospitalario son elevados y repercuten negativamente sobre su salud. ${ }^{29}$

Los principales síntomas que se presentan asociados a situaciones de estrés son los trastornos músculo-esqueléticos, depresivos y alteraciones psicosomáticas y el Burnout ${ }^{30}$.

El número de estudios realizados en personal de enfermería es superior a los que se han llevado a cabo en médicos, lo que parece indicar una mayor preocupación o interés de este colectivo por los riesgos psicosociales derivados de situaciones de estrés en el ámbito laboral.

En el estudio realizado por Dendaas ${ }^{20}$ mediante un test de congruencia ambiental realizado en personal de enfermería, concluye que las características del empleo o ambiente físico como el mal diseño del puesto, las condiciones ergonómicas precarias o la dificultad del ámbito de trabajo para generar buenas relaciones entre compañeros, contribuían negativamente favoreciendo un entorno laboral más estresante.

Como señalan Virtamen $^{15}$ y Dorevich $^{30}$, además de las áreas más estresantes definidas por Shaherl $^{54}$, algunas situaciones especiales de tensión y responsabilidad a las que se puede enfrentar el personal sanitario, como tener que abordar situaciones de emergencia o epidemias, que pueden alterar las relaciones interprofesionales e incrementar el tiempo de dedicación, pueden comportarse como agravantes de un ámbito laboral y repercutir incrementando más los niveles de estrés y las patologías asociadas.

Estudios realizados por Dorevitch ${ }^{30}$ sobre los riesgos laborales a los que se encuentran sometidos los médicos de emergencias, señalan el estrés emocional como uno de los principales peligros inherentes a la profesión y lo relacionan con una mayor posibilidad de asociación al Burnout.

Por otra parte, encontramos diferentes niveles de exposición al estrés en distintas especialidades, áreas o servicios del hospital ${ }^{30-32}$. De esta forma, Shaberl ${ }^{54}$ diferencia entre áreas estresantes y no estresantes siguiendo una serie de características demográficas y organizativas del trabajo en las que además se tienen en cuenta la edad, la experiencia, el género, el trabajo a turnos y la carga de trabajo.

Al comparar los resultados de diferentes estudios y autores, encontramos variaciones geográficas en cuanto a los efectos producidos sobre las poblaciones estudiadas. Estudios realizados por $S_{\text {metl }}{ }^{33}$ encontraron niveles similares de estrés entre los profesionales japoneses, chinos y españoles. Estos datos también se encuentran respaldados por los resultados encontrados por Kawakam ${ }^{34}$ al comparar grupos de profesionales de Japón con los de los países del sur de Europa.

La mayoría de los métodos utilizados en la evaluación de los riesgos psicosociales, se encuentran basados en las teorías de Karasek ${ }^{37}$ y Siegrist $^{39}$ (JCQ,ERI), los cuales presentan un elevado nivel de fiabilidad abalado por estudios de validación previos ${ }^{4}$, no existe un consenso internacional en el método de evaluación, si bien, los más utilizados son el Job Content Questionnaire, Effort Reward Imbalance y Nursing Stress Scale.

El apoyo social y las características individuales son moderadores de los riesgos psicosociales, lo que se encuentra descrito en diferentes trabajos incluidos en esta revisión. Voltmerl ${ }^{35}$ señala que el mantener buenas relaciones personales y profesionales entre colegas, participación en foros profesionales en la red, el mantener relaciones de pareja, grupos de amistad y de apoyo, podrían contribuir a la prevención de situaciones como Burnout y la aparición de diferentes patologías o síntomas derivadas de las situaciones de estrés.

Podemos concluir que la mayoría de estudios revisados encuentran un elevado nivel de estrés en el personal sanitario de hospitales y este nivel es mayor cuando el trabajo se 
desempeña en áreas como urgencias, oncología, anestesia y cuidados intensivos. En circunstancias especiales de mayor carga de trabajo, responsabilidad, fatiga o situaciones de emergencia, los niveles de estrés se incrementan de forma considerable.

Los niveles de estrés y afectación de los profesionales españoles podría compararse al de países como Japón y China, siendo más elevados que en otros países del entorno de la Unión Europea.

Teniendo en cuenta que la mayoría de los autores coinciden en que los ambientes laborales estresantes terminan provocando algún tipo de alteración física y psicológica en el personal sanitario del hospital, convendría realizar un análisis más exhaustivo de nuestro entorno, centrándose en el personal médico del ámbito hospitalario, por tratarse de una profesión que parece encontrarse sometida a elevados niveles de estrés, apenas se han encontrado estudios que aborden el tema y nos permitan conocer suficientemente la situación. Más aún cuando parece que nos encontramos entre los países de mayor nivel de riesgo para el estrés, como pueden ser Japón o China y con niveles más alejados de los que se encuentran en otros países del norte de Europa.

Desde el punto de vista práctico el conocimiento de los riesgos psicosociales en general y del nivel de estrés en particular junto con sus posibles características y peculiaridades son necesarias como base para una intervención sobre estos y la toma de medidas preventivas eficaces. ${ }^{9}$

En este sentido encontramos algunas iniciativas y recomendaciones institucionales orientadas hacia la prevención de situaciones favorecedoras de estrés en el ámbito profesional, como puede ser y a título de ejemplo, la nota técnica El estrés: Proceso de generación en el ámbito laboral, del Instituto Nacional de Seguridad e Higiene del Trabajo (NT 318, INSHT), pero aún parece que queda mucho camino por recorrer, no sólo en cuanto al conocimiento de la situación laborar en nuestro país y sus repercusiones, sino también a establecer medidas eficaces de prevención de riesgos relacionados con ambientes estresantes como en la prevención de patologías derivadas en los profesionales sanitarios que desarrollan su actividad en el ámbito hospitalario. Pese a esta necesidad percibida a la luz de los hallazgos encontrados, llama la atención como el número de publicaciones abordando este tema se encuentra con tendencia descendente desde 2009 hasta 2012 como se deduce de los datos presentados en la tabla II.

\section{REFERENCIAS BIBLIOGRÁFICAS}

1. Mingote Adán C y Antón A. El Estrés del médico. Ed. Díaz de Santos. 1999. ISBN: 84-7989-407-5.

2. Mingote Adán C, Moreno B y Galvez M. El desgaste del médico. Ed. Díaz de Santos. 2009. ISBN: 978-847978-919-0

3. Mingote Adán C y Gálvez Herrer M. El estrés del médico. masdermatología. 2011; 15:16-19. Doi:10.5538/18875181.2011.15.16

4. Wu H, Zhao Y, Wang JN, Wang L. Factors associated with occupational stress among Chinese doctors: a cross-sectional survey. Int Arch Occup Environ Health. 2010 Feb;83(2):155-64. doi: 10.1007/s00420-0090456-z. Epub 2009 Aug 22.

5. Roman-Hernandez. S.estres y burnout en profesionales de la salud de los niveles primarios y secundarios de atención.Rev. Cubana Sal Pub2003:29(9):3281.8.

6. Dorevitch, Samuel / Forst, Linda Hazard of emergency physicians The American Journal of Emergency Medicine, 18 (3), p.300-311, mayo 2000

7. Dendaas N.Environmental congruence and work-related stress in acute care hospital medical/surgical units: a descriptive, correlational study. HERD. 2011 Fall;5(1):23-42

8. Virtanen, Marianna / Kurvinen, Tiina / Terho, Kirsi / Oksanen, Tuula / Peltonen, Reijo / Vahtera, Jussi / Routamaa, Marianne / (...) / Kivimäki, Mika, Medical care Work hours, work stress, and collaboration among ward staff in relation to risk of hospital-associated infection among patients., 47 (3), p.310-318, Mar2000 
9. Shaher H, Ali A.Comparing Jordanian nurses jod stressors in stressful and non- stressful clinical areas. Contemporary Nurse (2011)37(2):173-187

10. Cevik, Arif Alper / Holliman, C James / Yanturali, Sedat, Burnout and emergency physicyans. Ulusal travma he acil cerrahi Dergisi journal = turco de la cirugía traumatológica y de emergencia: TJTES, 9 (2), p.85-89, 042003

11. Oates, R. K. / Oates, P., Stress and mental health in neonatal intensive care units.Archives of Disease in Childhood - Fetal and Neonatal Edition, 72 (2), p.F107-F110, Mar 1995

12. De Smet, P / Sans, S / Dramaix, M / Boulenguez, C / de Backer, G / Ferrario, M / Cesana, G / (...) / Kornitzer, Gender and regional differences in perceived job stress across Europe.M, European journal of public health, 15 (5), p.536-545, Oct 2005

13. Kawakami, Norito / Haratani, Takashi / Kobayashi, Fumio / Ishizaki, Masao / Hayashi, Takeshi / Fujita, Osamu / Aizawa, Yoshiharu / (...) / Araki, Shunichi, Occupational class and exposure to job stressors among employed men and women in Japan.Journal of epidemiology / Japan Epidemiological Association, 14 (6), p.204-211, Nov 2004

14. KaraseK K, Brisson C, Kawakami N, Houtman I, Bongers P, Amick B. The Job Content Questionnaire (JCQ): an instrument for internationally comparative assessments of psychosocial job characteristics

15. Siegrist J. Adverse health effects of high-effort/low-reward conditions. J. Occupational Healt Psycohology 1996, Vol 1, No 1, 27-41

16. Voltmer, Edgar / Spahn, Claudia Social support and physicians' health., Zeitschrift für Psychosomatische Medizin und Psychotherapie, 55 (1), p.51-69, Jan 2009

17. Albini E, Zoni S, Parrinello G, Benedetti L, Lucchini R. An integrated model for the assessment of stressrelated risk factors in health care professionals. Industrial Health. 2010; 49:15-23.

18. Diaz A, Feldman 1. Validación preliminar del cuestionario de estrés laboral (desbalance-esfuerzo/ recompensa) en una muestra de trabajadores de la salud venezolanos. Ciencia \& trabajo $2010 \mathrm{~N}^{\circ} 36320-326$.

19. Palacios ME, Paz MP. Estrés y manifestaciones psicosomáticas en médicos de hospitales de la ciudad de México. $1^{\circ}$ Congreso Internacional y $4^{\circ}$ Foro de las Américas en Investigación sobre factores Psicosociales, Estrés y Salud Mental en el Trabajo. Oct. 2012. Bogotá.

20. Valecillo M, Quevedo AL, Lubo A, Dos Santos A, Montiel M, Camejo M et al. Síntomas musculoesqueléticos y estrés laboral en el personal de enfermería de un hospital militar. Salud de los trabajadores 2009 v. 17 $\mathrm{N}^{\mathrm{o}} 285-95$.

21. Colunga-Rodriguez AC, Enriquez-Hernandez CB, Angel-Gonzalez M, Dominguez-Diaz R. Desarrollo científico enfermero. 2009 ago. $\mathrm{N}^{\circ}$ 17(7).

22. Choobineh A, Movahed M, Tabatabaie SH, Kumashiro M. Perceived demands and musculoskeletal disorders in operating room nurses of Shiraz city Hospitals. Industrial Health. 2010: 48: 74-84.

23. Arimura M, Imai M, Okawa M, Fujimora T, Yamada N. Sleep, mental heath status, and medical errors among Hospital nurses in Japan. Industrial Health .2010; 48: 811-817.

24. Wu SY, Li HY, Tian J, Zhu W, Li J, Wang XR. Health-related quality of life and its main related factors among nurses in China. Industrial Health. 2011: 49: 158-165..

25. Mehrad R, T Dennerlein JT, Haghighat M, Aminian O. Association between psychosocial factors and musculoskeletal symptoms among Iranian nurses. American Journal of Industrial Medicine. 2010; 53:1032-1039.

26. Mc Carthy VJC, Power S, Greiner BA. Perceived occupational stress in nurses working in Ireland. Occupational Medicine. 2010; 60: 604-610.

27. Warming S, Precht DH Suadicani P, Ebbehoj NE.Musculoeskeletal complaints among nurses related to patient handling tasks and psychosocial -based on logbook registrations.Applied Ergonomics. 2009; 40: 569-576.

28. Espinoza Guerrero, Marianela, Valverde hidalgo, Sharon, Vindas Salazar Hellen. Holistic experience of stressing nursing professionals. Rev.enfermeria Actual en Costa Rica (en Línea) 2011. $\mathrm{n}^{\circ} 20$

29. Kawasaki, Kazuyoshi / Sekimoto, Miho / Ishizaki, Tatsuro / Imanaka, Yuichi, Work stress and workload of full-time anesthesiologists in acute care hospitals in Japan. Journal of anesthesia, 23 (2), p.235-241, Jan 2009

30. Bagaajav, Ariunsanaa / Myagmarjav, Sugarmaa / Nanjid, Khuderchuluun / Otgon, Saranchuluun / Chae, Young Moon.Burnout and job stress among mongolian doctors and nurses., Industrial health, 49 (5), p.582-588, Jan 2011

31. Wang LJ, Chen CK, Hsu SC, Lee SY, Wang CS, Yeh WY.Active job, healthy job? Occupational stress and depression among hospital physicians in Taiwan. Ind Health. 2011; 49(2):173-84. Epub 2010 Dec 16. 
32. María Pilar López Layos, Gemma Torrejón Mora, Antolín Martín Marcos, Jaime Martín Morcillo, Francisco Pleite Gómez, María Angustias Torres Alaminos Estrés y satisfacción laboral de las enfermeras de hospitales toledanos. Metas de enfermería, ISSN 1138-7262, Vol. 14, №. 8, 2011 , págs. 8-14

33. BathiaN, KishoreJ, Anand T, Jiloha RC Occupational stress Amongst Nurses of two Tertiary Care hospitals in Delhi. AMJ 2010, 3, 11,731-738.

34. Monalisa de Cássia Fogaça,Werther Brunow de Carvalho,Vanessa de Albuquerque Cítero,Luiz Antonio Nogueira-Martins.Estudio preliminar sobre el estrés ocupacional de médicos y enfermeros en UTI pediátrica y neonatal: el equilibrio entre

Esfuerzo y recompensa Ver latino-americana enfermagen 18(1) (07 pantallas) ene-feb-2010

35. Tsutsumi A, Iwata N, Watanabe N, de Jonge J, Pikhart H, Fernández-López JA, Xu L, Peter R, Knutsson A, Niedhammer I, Kawakami N, Siegrist J.Application of item response theory to achieve cross-cultural comparability of occupational stress measurement. Int J Methods Psychiatr Res. 2009; 18(1):58-67.

36. Lindo JL, Lagrenade J , McCaw-Binns A, Eldemire-D Shearer Estado de salud y las prácticas de mantenimiento de la salud entre médicos y enfermeras en dos hospitales de Jamaica. West Indian J. Med. 2009 DEC; 58 (6):539-45.

37. Hamaideh SH , Ammouri A .Comparing Jordanian Nurses' job stressors in stressful and non-stresful clinical areas. Contemporary Nurses. 2011 Feb; 37 (2):173-87. Doi: 10.5172/conu.2011.37.2.173.

38. Wells, John / Denny, Margaret / Cunningham, Jennifer , 2011Work related wtress and European policy A comparative exploration of contextual stressors in the rehabilitacion sector in five European countries Journal of mental Health 20 (2), p.165-173, abril

39. Elizabeth Dougherty, Et al.Factors Associated whith work stress and Professional Satisfaction in oncology staff.American journal of hospice \& palliative medicine26(2)2009 105-111

40. Comité mixto OIT/OMS. Identificación y control de los factores psicosociales nocivos en el trabajo. Ginebra 1984

41. $2 .^{\circ}$ Foro de las Américas en Investigación sobre factores psicosociales. Chile 22-28 octubre 2008.

42. Mas R, Escribá V. La versión castellana de la escala "The nursing stress scale" Proceso de adaptación transcultural. Rev.Esp. Salud Pública vol.72 nº 6 529-538.

43. Wollfgang AP. The healt professions stress inventory. Psycological Reports 1998, 62, 220-222.

44. Ros S, Comas A, Garcia-Garcia M. Validación de la versión española del cuestionario PHQ-15 para la evaluación de síntomas físicos en pacientes con trastornos de depresión y/o ansiedad: estudio DEPRESOMA. Actas Españolas de Psiquiatría. 2010, Dic, 38 (6):345-357.

45. Golberg D. Manual of the general health questionnaire- 28 (GHQ-28). Windsor: NFER- Nelson. 1978.

46. Aranaz J, Mira J. Cuestionario Font Roja. Un Instrumento de medida de la satisfacción en el medio hospitalario. Todo Hospital 1988, 52: 63-6.

47. Blegen MA. Nurses`job satisfaction: a meta- analysis of related variables. Nur Res. 1993. 42(1): 36-41.

48. Zangaro CA, Soeken KL. A meta-analysis of studies nurses job satisfaction. Res Nurs Health 2007 30(4): $445-58$.

49. Enfoque multidimensional y transversal, Peiró 2001-2008

50. ILO Introductory report: global trends and challenges on occupational safety and healt. XIX World Congress on Safety and Health at Work: Istanbul Turkey, 11-15 sep. 2011.

51. Joensuu M, Vaananen A, Koskinen A, Kivimaki M, Virtanen M, Vathera J. Psychosocial Word environment and hospital admissions due to mental disordes: a 15-year prospective study of industrial employees. J Affect DISORD. 2010 Jul; 124 (1-2): 118-25.

52. Aldana SG. Financial impact of health promotion programs: a comprehensive review of the literature. Is J Health Promot? 2001 May-Jun; 15(5):296-320.

53. Entornos laborales saludables: fundamentos y modelo de la OMS. Contextualización, Prácticas y literatura de apoyo. 2010 6(6) 53-56.

54. Institute for Work \& Health. Are workplace prevention programs effective? Sharing best evidence: highlights of a systematic review. Ago. 2008.

55. Niveles de evidencia del Scottish Intercollegiate Guidelines Network (SIGN) www.sign.ac.uk/guidelines

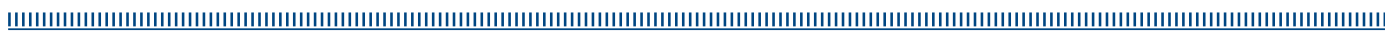

